\title{
New imaging opportunities at the DIAMOND beamline I13L
}

C. Rau, D. Batey, S. Cipiccia, X. Shi, S. Marathe, et al.

C. Rau, D. Batey, S. Cipiccia, X. Shi, S. Marathe, M. Storm, A. Bodey, M. Zdora, "New imaging opportunities at the DIAMOND beamline I13L," Proc. SPIE 11112, X-Ray Nanoimaging: Instruments and Methods IV, 111120L (1 October 2019); doi: 10.1117/12.2543799

SPIE Event: SPIE Optical Engineering + Applications, 2019, San Diego, California, United States 


\title{
New Imaging Opportunities at the DIAMOND Beamline I13L
}

\author{
C. Rau* ${ }^{1,2,3}$, D. Batey ${ }^{1}$, S. Cipiccia ${ }^{1}$, X. Shi ${ }^{1}$, S. Marathe ${ }^{1}$, M. Storm ${ }^{1}$, A. Bodey ${ }^{1}$ and M. Zdora ${ }^{1,4,5}$ \\ ${ }^{1}$ Diamond Light Source Ltd, Harwell Science and Innovation Campus, Didcot, OX 11 0DE, UK \\ ${ }^{2}$ University of Manchester, School of Materials Grosvenor St., Manchester, M1 7HS, UK \\ ${ }^{3}$ Northwestern University, 303 E. Chicago Avenue, Chicago, IL 60611-3008, USA \\ ${ }^{4}$ Department of Physics \& Astronomy, University College London, London WC1E 6BT, UK \\ ${ }^{5}$ Department of Physics \& Astronomy, University of Southampton, Southampton, SO17 1BJ, UK
}

\begin{abstract}
The Diamond Beamline I13L is dedicated to micro- and nano- imaging, with two independently operating branchlines. The imaging branch preforms imaging in real space, with In-line phase contrast imaging and grating interferometry at micrometre resolution and full-field transmission microscopy up to $50 \mathrm{~nm}$ spatial resolution. Highest spatial resolution is achieved on the coherence branchline, where diffraction imaging methods such as Ptychography and Bragg-CDI are performed. The article provides an update about the experimental capabilities at the beamline with an emphasis on the rapidly evolving ptychography capabilities. The latter has evolved to an user-friendly method with non-expert users able to explore their science without any specific a-priory knowledge.
\end{abstract}

Keywords: tomography, synchrotron radiation, nano-imaging, phase contrast, X-ray microscopy, ptychography, CDI

\section{INTRODUCTION}

Synchrotron radiation provides unique opportunities for high-resolution imaging and exploration of new science. The high photon flux gives access for in-situ and in-operando studies with dedicated sample environments. High spatial resolution data can be recorded with reasonable time scales. The high brilliance of the source and the coherence of the light are explored for imaging in real and reciprocal space. The energy tuneability of the radiation gives access to chemical information.

The Diamond beamline I13L addresses science on the micro- and nano- lengthscale for samples ranging from some tens of microns to millimeter size. While micro-tomography is well established at the beamline and accessible for any application, high-resolution experiments have made significant progress in the recent past. In particular the experiment for ptychography is now available with a complete data pipeline for non-expert users. The emphasis is on the usability of the instrument to a wide range of scientific applications and a large user community.

\section{THE BEAMLINE}

The I13 beamline is an imaging beamline located in one of the long straight sections of the Diamond $3 \mathrm{GeV}$ electron storage ring[1, 2]. The long straight section has been modified by the so-called 'mini-beta' layout, dividing the section in two shorter units with smaller vertical electron beam. Each section operates independently an in-vacuum undulator with ID gaps down to $5 \mathrm{~mm}$. The design provides about one order of magnitude increased photon flux at higher photon energies compared to the unmodified layout. The horizontal beta function of the electron beam is shaped to form a (virtual) focus downstream of the location of the insertion device (ID). For the coherence branch the focal spot size is about $200 \mu \mathrm{m}$ at a

\footnotetext{
X-Ray Nanoimaging: Instruments and Methods IV, edited by Barry Lai, Andrea Somogyi,

Proc. of SPIE Vol. 11112, 111120L · C The Authors. Published under a Creative

Commons Attribution CC-BY 3.0 License · doi: 10.1117/12.2543799
} 
distance of $15 \mathrm{~m}$ from the ID. This location in the front end is accessible and a set of slits allows adjustment of the (virtual) source size and therefore the lateral coherence length to the requirements in the experimental hutch at a distance over $200 \mathrm{~m}$ from the source. The first optics hutch hosts further slits for beam trimming, a container for filters with a large variety of combinations. On the coherence branch a set of compound refractive lenses (CRLs) is used for changing the beamsize in the external hutch. Each branch deflects the beam horizontally with mirrors, different stripes on these mirrors define the cut-off energy at higher photon energies. The mirror on the imaging branchline is bendable to horizontally collimate/focus the beam, the coherence branch mirror is plane and has an undercut to compensate for the heatload and to preserve the surface flatness of about 150nrad. On the imaging branch a multilayer Laue monochromator (MLM) with different multilayer systems is used, ranging from 1-5\% bandpass. In the external building a double crystal monochromator (DCM) with Si(111) crystals on the imaging branch and a four-bounce monochromator (QCM) in horizontal deflecting mode are installed. All beamline components are water-cooled.

\section{EXPERIMENTAL FACILITIES AT I13}

\subsection{The Diamond-Manchester Imaging branchline}

The imaging branch covers the micron to 50nm resolution range. The micro-tomography setup provides micrometer resolution, limited by the detector system which includes a visible light microscope optic. Tomographic scans can be performed within seconds. The features of weakly absorbing samples can be enhanced with the In-line phase contrast method, moving the detector up to two meters from the sample. Grating interferometry is currently implemented with the same spatial resolution, but providing small-angle scattering and quantitative phase information [3-5]. Finally a full-field microscope reaches $50 \mathrm{~nm}$ resolution over about $100 \mu \mathrm{m}$ field of view [6, 7]. The exposure time is down to some tens of $\mathrm{ms}$, similar to micro-tomography. For all methods broader energy bandwidth radiation is used typically, either with the socalled pink-beam (In-line phase contrast and Grating Interferometry) or the multilayer monochromator (MLM).

\subsection{The Coherence branchline}

The coherence branch is designed for experiments with extended coherence requirements, primarily CDI and ptychography (see figure 1) [8, 9]. A full ptychographic imaging pipeline is currently available for user operations, with the ongoing development of multi-modal (XRF, XRD) and Bragg geometries. At the experimental station the lateral coherence length in the vertical direction is of several hundred microns [10]. The horizontal coherence length can be adapted to match the vertical coherence length with slits located in the front end. Under these conditions the focusing optics at the endstation are fully coherently illuminated. The blazed Fresnel-Zone plates provide a minimal spot size of about 200nm. Larger beam spots are typically generated either by defocusing the optics or using zone plates with larger focal length.

The sample stage consists of a 6-axis base stack, with (from bottom to top) a vertical lift stage, an x-z translation stage, and $x-z$ tilt stage, an air bearing rotation stage (about y) on top of which sits on a 2-axis scanning stage, with an x-z stepper motor for alignment and an $x-y-z$ piezo stage. The scanning stage is able to scan in any plane, either relative to, or independent of the stages beneath -an important feature for both ptycho-tomography and Bragg ptychography. The piezo stage has a range of $300 \mu \mathrm{m}$ in all three directions. The air bearing stage has a run-out of $50 \mathrm{~nm}$. The data is recorded either in transmission or under Bragg conditions. The transmission detectors are supported on a linear rail of 3-14m and the Bragg detectors are placed on a robot arm at a nominal distance of $3 \mathrm{~m}$ and translates through a solid angle limited by $30^{\circ}$ in the vertical and horizontal directions [8, 9]. Because of the large flexibility and space available in the hutch, experimental conditions can be varied to cover a large range of resolutions and sample sizes, a detector -sample distance typically ranging from 3.5 to $14 \mathrm{~m}$ with corresponding effective pixel size of 4 and 16nm respectively [11, 12]. 


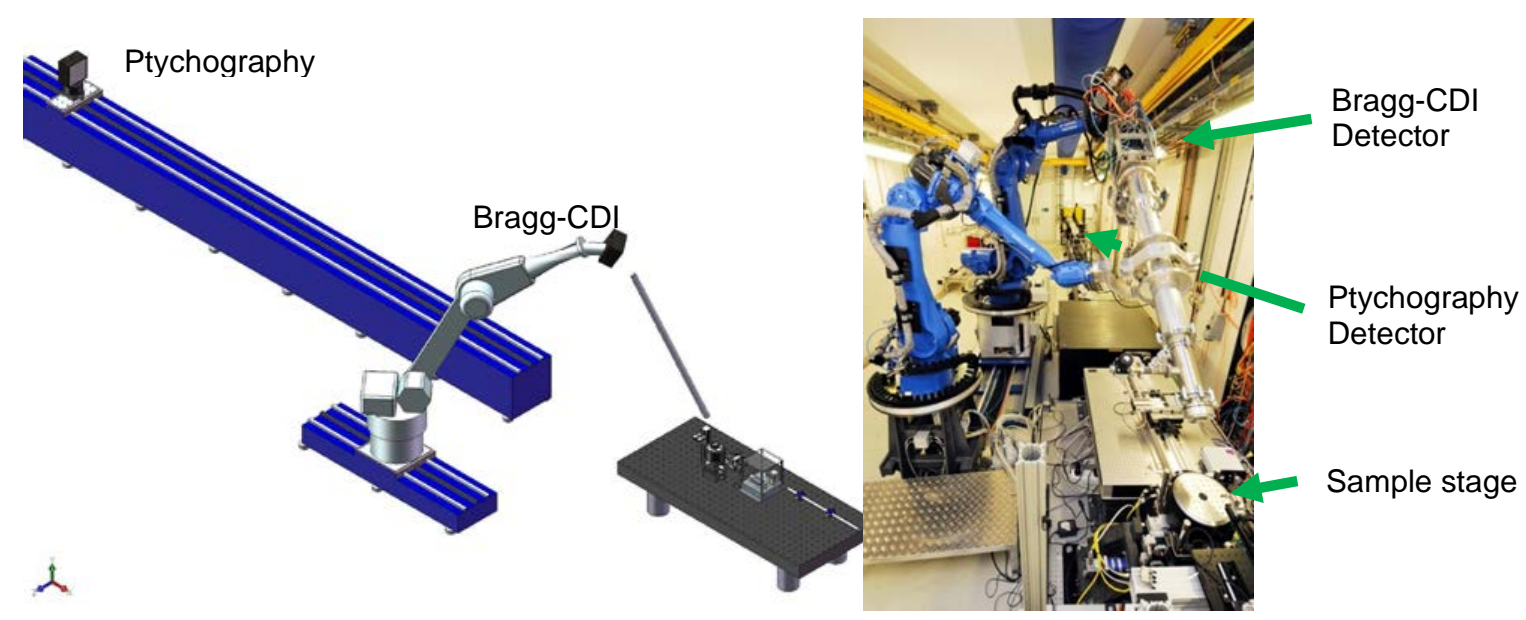

Figure 1: Left: Scheme for experimental setup on coherence branch. Right: Image of experimental hutch. In foreground sample stage, robot arm and detector for Bragg CDI. In the far background at the end of the hutch the ptychography detector is located.

\subsubsection{Recording of data}

The data is recorded with the in-house developed EXCALIBUR detector [13, 14], containing a 6x8 MediPix3 array with $100 \mathrm{~Hz}$ recording speed in continuous mode and $1 \mathrm{kHz}$ in burst mode. For fast recording an Eiger 500k detector is being commissioned (more details below). The smaller version of the EXCALIBUR is the commercially available Merlin detector, using a 2x2 MediPix3 array. The MediPix3 has a pixel size of 55um, and has an improved radiation hardness compared to its predecessors.

Spectroscopic capabilities are added - but not currently implemented permanently - with the Vortex detector which can be operated with a multi-element readout EXPRESS3 module.

We recently demonstrated the possibility of element specific ptychography from a single scan using the hyperspectral SLcam detector (see figure 2) $[15,16]$. The device is a pnCCD with 48um side length pixels in a 264x264 array. The signal depth is 11 bit with an energy resolution of about $150 \mathrm{eV}$. For the experiment the energy width of the undulator harmonic was increased to permit the single scanning of data into the different energy channels of the SLcam.

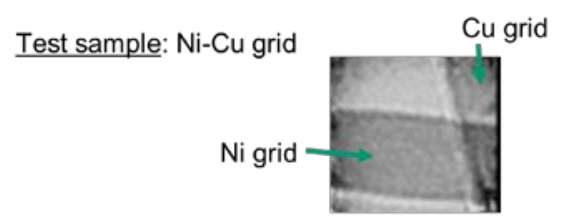

Single detector scan:

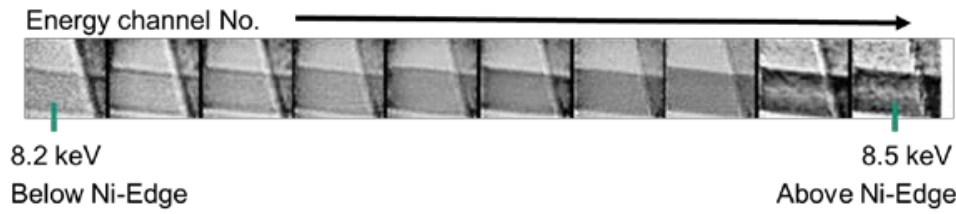

Figure 2: Image series extracted from single ptychographic scan using energy selective SLcam hyper-spectral detector. Test sample consists of a stacked copper and nickel grid, the latter can be identified by its absorption edge [16]. 


\subsubsection{Recording speed}

Currently data is recorded in standard operation with some tens of $\mathrm{Hz}$ rate, with scanning area of $20 \mu \mathrm{m}^{2} \mathrm{~s}^{-1}$. A typical 3D ptycho-tomographic scan requires ten hours of recording time, recording up to 1000 projection angles.

We have demonstrated that we are capable to reconstruct ptychographic data with $10 \mu$ s exposure time using pink beam, which leads to a possible recording speeds in excess of $10 \mathrm{kHz}$ (see figure 3) [11]. Detector, stages and beamline controls are currently upgraded to fully explore the opportunity of rapid scanning.
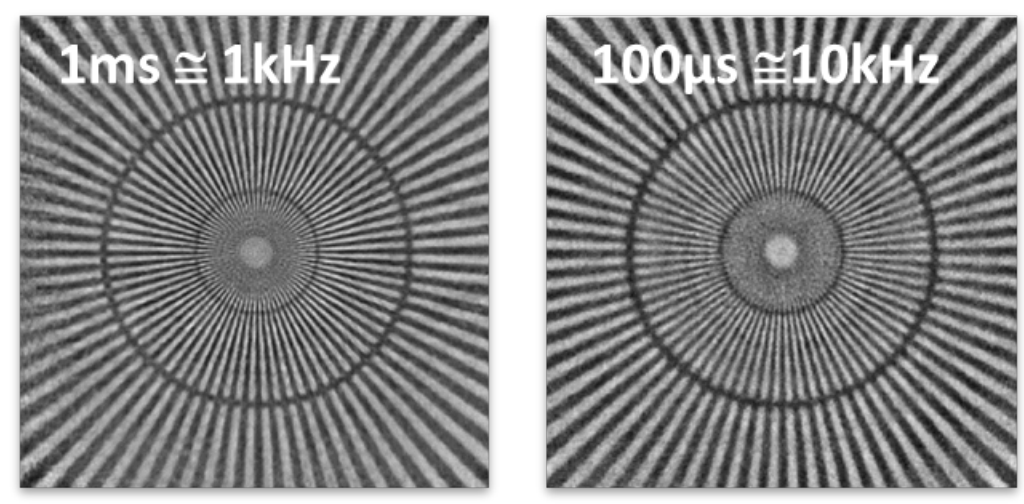

Figure 3: Reconstructed data of Siemens star with short exposure times, left 1ms, right $100 \mu \mathrm{s}$. The latter proofs the feasibility of scanning at $10 \mathrm{kHz}$ rate. The two circular indicator lines are positioned at spoke separations of 200 and 100nm [11].

The detector has been upgraded to an Eiger 500k with the capability of $9 \mathrm{kHz}$ data acquisition rate at 4 bit image depth and $3 \mathrm{kHz}$ in 12 bit mode. The rapid sample scanning is operated with software originally designed for serial crystallography $[17,18]$ with low-level programming of the beamline's PMAC controller and a user-friendly GUI interface. For the future it is planned to succeed the software with the Diamond developed Malcolm/mapping architecture.

The recording at high-speed will occur in fly scan mode and is currently tested. More recently we managed to increase the scanning speed in fly-scan mode to $20 \mu^{2} \mathrm{~s}^{-1}$ with an acquisition rate of $600 \mathrm{~Hz}$. The in-house developed PtyREX ptychographic software handles the necessary adjustments for sample position and the polychromaticity of the radiation spectrum (pink beam).

\subsubsection{Beamline controls, data analyses and the PtyREX software package}

For the I13 coherence branchline a GUI interfaced software package has been implemented to control the experiment and to analyse the data (interface screenshot see figure 5). For reconstructing the ptychographic data the PtyREX software package has been developed. The code is based on the ePie algorithm [19] aiming for iterative Ptychographic Reconstruction for electrons and X-rays, as well as visible light. The software is modular and has a multitude of options for reconstructing the data. The beam module can retrieve the probe under partial spatial coherence but also limited temporal coherence, when multiple wavelengths are used for the experiment. In the scanning module the positions of the scanning points can be corrected, which is essential when recording fly-scans [20]. Regarding the detector, the data is scaled with the detector response function and data can be eventually up-sampled [21, 22]. Super-resolution depending on the experimental conditions can be achieved. The program can be executed with any permutations of the modules. 
The python-written code is stored on GitHub and is available on request [23]. The beamline produces data in either tiff or HDF format with a NeXus header file. Other reconstruction packages are also being available, such as PtyPy [24, 25].

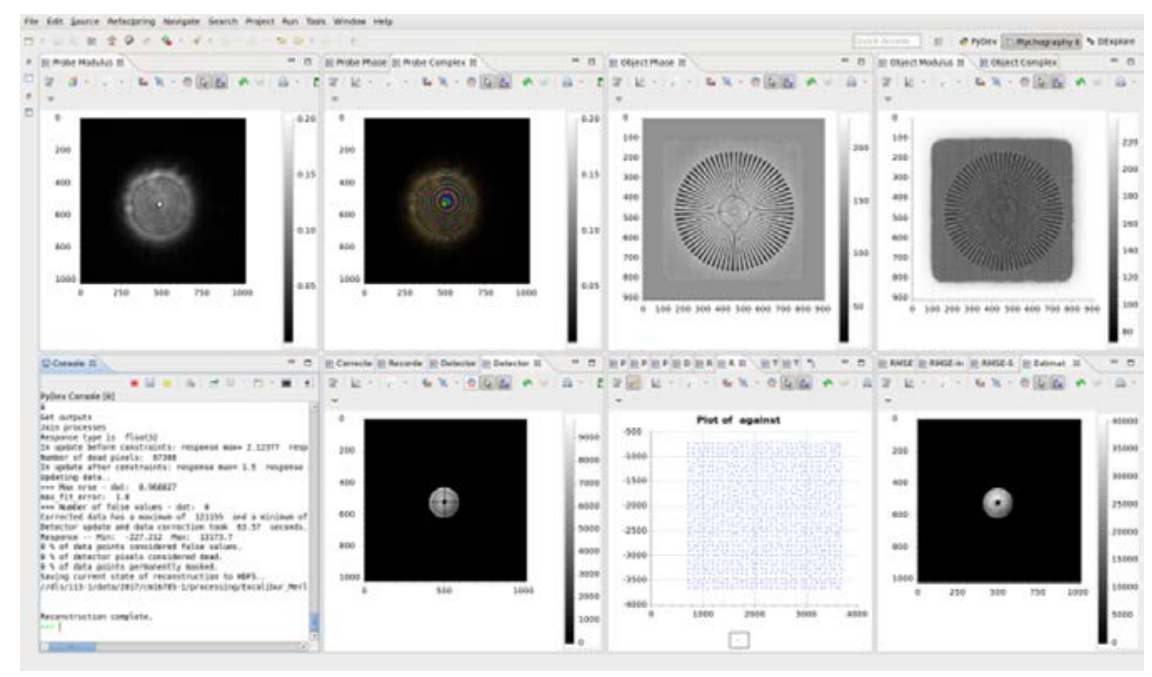

Figure 4: The controls and reconstruction software is fully integrated into the Diamond GDA/DAWN architecture and provides a complete data recording and analyses chain [23].

\section{SCIENCE COVERED}

One example for multi-scale and multi-modal research is the investigation on fall-out particles collected from the Fukushima reactor accident (see figure 5) [26]. The micro-tomographic data shows the formation of gas bubbles embedded in a glassy structure. Both indicate that a sudden burst at high temperatures must have happened during the incident (glasses form during sudden changes of exterior conditions). The co-location of chemical elements in the particle provides a scenario for the fire following the burst and where and what caught fire. The element specific fluorescence information is revealed by spectro-ptychographic mapping.
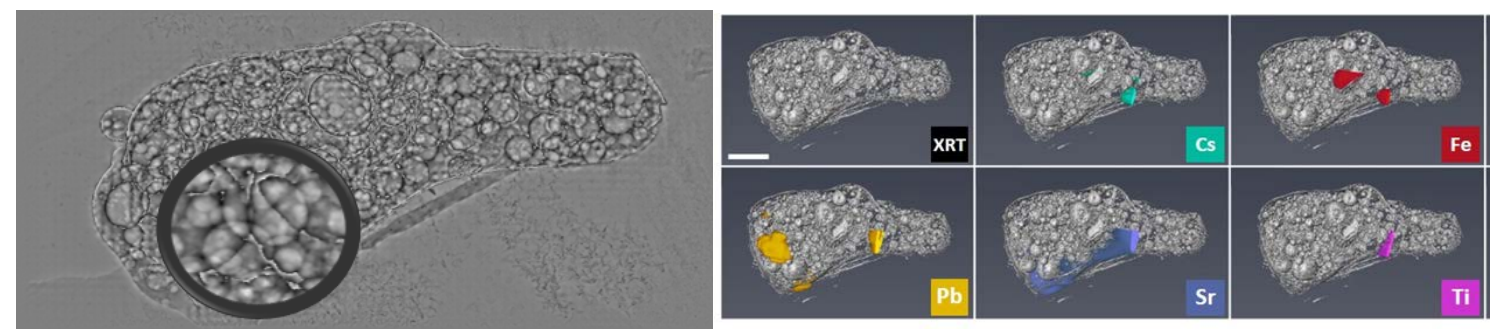

Figure 5: Example of multi-scale and multi-modal imaging; study of fall-out particle from Fukushima accident. Left: ptychographic reconstruction of single projection. Right: 3D rendering of micro-tomogaphic data (upper left) and correlation of different elements detected by fluorescence [26].

Other examples for multi-scale imaging at I13 are batteries research (charge cycles, electrode materials), biological and bio-medical applications (brain research, study of insects, imaging of chromosomes) and materials sciences (crack formation and propagation, material corrosion, dendritic crystal growth). 


\section{SUMMARY}

The Diamond beamline I13L is dedicated to multi-scale and multi-modal imaging. Two independently operating branches cover the micro- and nano-lengthscale. The imaging branch offers In-line phase contrast imaging, Grating interferometry and full-field microscopy covering the micron to 50nm. Ultimate resolution is achieved with Ptychography and BraggCDI. In particular Ptychography is implemented now as a user-friendly experiment for non-experts, user-friendly BraggCDI is to be developed secondly. We demonstrated the feasibility of recording data with $10 \mathrm{kHz}$ rate and element-specific recording in a single scan. The PtyREx software is used for data analyses and includes modules for position correction and polychromaticity. The experiment has now evolved to a user-friendly package, suitable for non-expert scientists in ptychography. We currently develop the science exploiting the instrumental capabilities offered by the beamline.

\section{ACKNOWLEDGEMENTS}

The Diamond Manchester collaboration is acknowledged. The I13 team closely collaborates with C. David and Florian Döring (PSI) for X-ray optics. Kaz Wanelik, Andy Wilson and Ben Bradnick provide excellent support for the beamline controls and data acquisition. Special thanks the colleagues from the Diamond I24 beamline (Robin Owen) for their help to improve ptychographic scanning at our beamline.

\section{REFERENCES}

[1] Rau, C., Wagner, U., Pesic, Z., and De Fanis, A., “Coherent imaging at the Diamond beamline I13,” Physica Status Solidi a-Applications and Materials Science, 208(11), 2522-2525 (2011).

[2] Rau, C., Wagner, U. H., Vila-Comamala, J., Bodey, A., Parson, A., Garcia-Fernandez, M., De Fanis, A., and Pesic, Z., "Imaging in Real and Reciprocal Space at the Diamond Beamline I13," AIP Conference Proceedings, 1696, 020047.

[3] Marathe, S., Zdora, M. C., Zanette, I., Cipiccia, S., and Rau, C., "Comparison of Data Processing Techniques for Single Grating X-ray Talbot Interferometer Data," Proceedings of SPIE, 10391.

[4] Teh, I., McClymont, D., Zdora, M. C., Whittington, H. J., Davidoiu, V., Lee, J., Lygate, C. A., Rau, C., Zanette, I., and Schneider, J. E., "Validation of diffusion tensor MRI measurements of cardiac microstructure with structure tensor synchrotron radiation imaging,” Journal of Cardiovascular Magnetic Resonance, 19, (2017).

[5] Zdora, M. C., Vila-Comamala, J., Schulz, G., Khimchenko, A., Hipp, A., Cook, A. C., Dilg, D., David, C., Grunzweig, C., Rau, C., Thibault, P., and Zanette, I., "X-ray phase microtomography with a single grating for highthroughput investigations of biological tissue,” Biomedical Optics Express, 8(2), 1257-1270 (2017).

[6] Vila-Comamala, J., Bosgra, J., Eastwood, D. S., Wagner, U., Bodey, A. J., Garcia-Fernandez, M., David, C., and Rau, C., "Transmission X-ray Microscopy at Diamond-Manchester I13 Imaging Branchline," AIP Conference Proceedings, 1696, 020036 (2016).

[7] Storm, M., Cipiccia, S., Marathe, S., Kuppili, V. S. C., Doring, F., David, C., and Rau, C., "The Diamond I13-2 Transmission X-ray Microscope: Current Status and Future Developments,” Microscopy and Microanalysis, 24(S2), 216-217 (2018).

[8] Pešić, Z. D., Wagner, U. H., and Rau, C., "Design of the experimental stage for coherent diffraction imaging," Diamond Light Source Proceedings, 1(SRMS-7), e103 (2010).

[9] Pesic, Z. D., De Fanis, A., Wagner, U., and Rau, C., "Experimental stations at I13 beamline at Diamond Light Source," Journal of Physics Conference Series, 425, 182003.

[10] Wagner, U. H., Parsons, A., Rahomaki, J., Vogt, U., and Rau, C., "Characterising the Large Coherence Length at Diamond's Beamline I13L," AIP Conference Proceedings, 1741, 050022 (2016).

[11] Cipiccia, S., Batey, D., Shi, X., Williams, S., Wanelik, K., Wilson, A., Martin, P., Scott, T., and Rau, C., "Multiscale multi-dimensional imaging at I13-coherence branchline in Diamond Light Source," AIP Conference Proceedings, 2054, 050005 (2019). 
[12] Sala, S., Kuppili, V. S. C., Chalkidis, S., Batey, D. J., Shi, X. W., Rau, C., and Thibault, P., "Multiscale X-ray imaging using ptychography,” Journal of Synchrotron Radiation, 25, 1214-1221 (2018).

[13] Tartoni, N., Dennis, G., Gibbons, P., Gimenez, E., Horswell, I., Marchal, J., Pedersen, U., Pesic, Z., Plackett, R., Rau, C., Somayaji, R., Spiers, J., Thompson, J., Willis, B., Angelsen, C., Booker, P., Burge, S., Lipp, J., Nicholls, T., Taghavi, S., and Thorpe, M., "Excalibur: a three million pixels photon counting area detector for coherent diffraction imaging based on the Medipix3 ASIC," IEEE Nuclear Science Symposium and Medical Imaging Conference, 530-533 (2012).

[14] Williams, S. J., Batey, D., Cipiccia, S., Angelsen, C., Crook, R., Gimenez, E., Horswell, I., Marchal, J., Nicholls, T., Pedersen, U., Taghavi, S., Tartoni, N., Rau, C., Thompson, J., Willis, B., and Ieee, "The ExcaliburRX-3M XRay Photon Counting Area Detector for Coherent Diffraction Imaging at the I13 Beamline at Diamond Light Source," IEEE Nuclear Science Symposium and Medical Imaging Conference.

[15] Scharf, O., Ihle, S., Ordavo, I., Arkadiev, V., Bjeoumikhov, A., Bjeoumikhova, S., Buzanich, G., Gubzhokov, R., Gunther, A., Hartmann, R., Kuhbacher, M., Lang, M., Langhoff, N., Liebel, A., Radtke, M., Reinholz, U., Riesemeier, H., Soltau, H., Struder, L., Thunemann, A. F., and Wedell, R., “Compact pnCCD-Based X-ray Camera with High Spatial and Energy Resolution: A Color X-ray Camera,” Analytical Chemistry, 83(7), 2532-2538 (2011).

[16] Batey, D. J., Cipiccia, S., Van Assche, F., Vanheule, S., Vanmechelen, J., Boone, M. N., and Rau, C., "Spectroscopic imaging with single acquisition ptychography and a hyperspectral detector," Scientific Reports, 9(1), 12278 (2019).

[17] Sherrell, D. A., Foster, A. J., Hudson, L., Nutter, B., O'Hea, J., Nelson, S., Pare-Labrosse, O., Oghbaey, S., Miller, R. J. D., and Owen, R. L., "A modular and compact portable mini-endstation for high-precision, high-speed fixed target serial crystallography at FEL and synchrotron sources,” Journal of Synchrotron Radiation, 22, 1372-1378 (2015).

[18] Owen, R. L., Axford, D., Sherrell, D. A., Kuo, A. L., Ernst, O. P., Schulz, E. C., Miller, R. J. D., and MuellerWerkmeister, H. M., "Low-dose fixed-target serial synchrotron crystallography," Acta Crystallographica Section DStructural Biology, 73, 373-378 (2017).

[19] Rodenburg, J. M., Hurst, A. C., Cullis, A. G., Dobson, B. R., Pfeiffer, F., Bunk, O., David, C., Jefimovs, K., and Johnson, I., "Hard-X-Ray Lensless Imaging of Extended Objects," Physical Review Letters, 98(3), 034801 (2007).

[20] Maiden, A. M., Humphry, M. J., Sarahan, M. C., Kraus, B., and Rodenburg, J. M., “An annealing algorithm to correct positioning errors in ptychography,” Ultramicroscopy, 120, 64-72 (2012).

[21] Edo, T. B., Batey, D. J., Maiden, A. M., Rau, C., Wagner, U., Pesic, Z. D., Waigh, T. A., and Rodenburg, J. M., "Sampling in x-ray ptychography," Physical Review A, 87(5), (2013).

[22] Batey, D. J., Edo, T. B., Rau, C., Wagner, U., Pesic, Z. D., Waigh, T. A., and Rodenburg, J. M., "Reciprocal-space up-sampling from real-space over sampling in x-ray ptychography,” Physical Review A, 89(4), 043812 (2014).

[23] Batey, D. J., "PtyREX: A user-friendly software package for reconstructing ptyhcographic data recorded with electrons, X-rays and visble light," (in preparation).

[24] Enders, B., and Thibault, P., “A computational framework for ptychographic reconstructions,” Proceedings of the Royal Society a-Mathematical Physical and Engineering Sciences, 472(2196), (2016).

[25] Sala, S., Batey, D. J., Prakash, A., Ahmed, S., Rau, C., and Thibault, P., "Ptychographic X-ray computed tomography at a high-brilliance X-ray source,” Optics Express, 27(2), 533-542 (2019).

[26] Martin, P. G., Louvel, M., Cipiccia, S., Jones, C. P., Batey, D. J., Hallam, K. R., Yang, I. A. X., Satou, Y., Rau, C., Mosselmans, J. F. W., Richards, D. A., and Scott, T. B., "Provenance of uranium particulate contained within Fukushima Daiichi Nuclear Power Plant Unit 1 ejecta material,” Nature Communications, 10, 2801 (2019). 